\title{
Dust tail of the distant comet C/1999 J2 (Skiff)
}

\author{
P. P. Korsun ${ }^{1,2}$ and G. F. Chörny ${ }^{1}$ \\ 1 Main Astronomical Observatory, Ukrainian Academy of Sciences, Kyiv, Ukraine \\ e-mail: korsun@mao.kiev.ua \\ 2 Isaac Newton Institute of Chile, Kiev Branch, Russia
}

Received 9 May 2003 / Accepted 7 July 2003

\begin{abstract}
CCD observations of comet C/1999 J2 (Skiff) were made at the Pik Terskol Observatory on September 15, 1999. The 2-m telescope equipped with the two-channel focal reducer of the Max-Plank-Institute for Aeronomy was used to study the cometary environment. In spite of the large heliocentric distance, $7.24 \mathrm{AU}$, a straight dust tail with fairly well defined boundaries was recorded. These data provide an opportunity to study the peculiarity of dust, that is not driven by water vapor. To fit the dust tail, a Monte Carlo model was developed. We trace the trajectories of about $10^{7}$ sample grains to construct the detailed brightness distribution in the comet tail. The simulated isophote field and the observed one agree very well. In our model we also take into account the heliocentric dependence of the dust production rate and ejection velocity, and the dust ejection anisotropy. To transform the particle population to brightness in the modelled tail we calculate the scattering cross section of a separate particle using Mie theory. The age, ejection velocity, dust size distribution, minimum and maximum size of the involved dust particles have been derived from the model giving the best fit. The intensity map is in agreement with a flow of the slowly travelling icy grains. It has been determined that the age of the dust tail of comet C/1999 J2 (Skiff) was, at the moment of the observations, about 540 days and that the main reason for its appearance is likely phase transition from amorphous to crystalline water ice activated at the heliocentric distance of 8.6 AU.
\end{abstract}

Key words. comets: individual: C/1999 J2 (Skiff)

\section{Introduction}

Comet C/1999 J2 (Skiff) was discovered by Skiff in the frame of the Lowell Observatory Near-Earth Object Search (LONEOS) program on May 13, 1999 (Skiff et al. 1999). In spite of the large heliocentric distance, 7.6 AU, the comet showed quite significant activity. A well condensed coma of diameter about $15^{\prime \prime}$ with a faint tail extending about $40^{\prime \prime}$ was seen at the moment of discovery. Further observations showed that the comet has a flattened light curve without any burst events (Shanklin 2001).

According to the well known formula for average variation of the apparent comet magnitude $m$ with heliocentric $r$ and geocentric $\Delta$ distances

$m=M_{0}+5 \log (\Delta)+2.5 n \log (r)$

the absolute magnitude $M_{0}$ for the comet is $1.5^{\mathrm{m}}$ when the activity parameter $n$ is 4 . This value of $M_{0}$ argues that the comet is a giant one. For example, the $M_{0}$ parameter is -0.8 for comet $\mathrm{C} / 1995 \mathrm{O} 1$ (Hale-Bopp) and 5.0 for comet 29P/Schwassmann-Wachmann. Comet C/1999 J2 (Skiff) passed its perihelion on April 5, 2000 at a heliocentric distance of 7.11 AU and the maximum brightness of $14.1^{\mathrm{m}}$ was reached

Send offprint requests to: P. P. Korsun, e-mail: korsun@mao . kiev. ua in May 2000. It should be noted that it is one of the largest perihelion distances among known comets.

Unfortunately, there is little analyzed data concerning this unique object. Fukushima et al. (2000) observed the comet shortly after its perihelion passage in late April, 2000 and detected a dust antitail. Analyzing CCD images obtained on 6 and 9 July, 2000, Szabo et al. (2001) found a rotation period of 0.96 day. A nuclear diameter of $10 \pm 8 \mathrm{~km}$ was derived assuming a 0.04 albedo. A slope of the logarithmic coma brightness within the 13.'0 aperture was about -1.6 , significantly larger than in an isotropic steady-state outflow case.

\section{Observations and data reduction}

CCD observations of comet C/1999 J2 (Skiff) were made at the Pik Terskol Observatory on September 15, 1999. The heliocentric and geocentric distances of the comet were 7.24 AU and 7.55 AU, respectively. Its phase angle was equal to 7.6 .

The 2-m telescope equipped with the 2-channel focal reducer of the Max-Planck-Institute for Aeronomy (Jockers 1997) was used to detect the comet's atmosphere in the blue and red spectral windows simultaneously. The observed sky fields were $7 ! 8 \times 7 ! 8$ with a pixel size of $11^{\prime \prime} 0$ and $7 ! 8 \times 5 ! 2$ with a pixel size of 0 ' $^{\prime} 8$ in the blue and red channels, respectively. To isolate the emissions of the $\mathrm{CN}$ molecules the interference 
Table 1. Log of observations.

\begin{tabular}{cccccccc}
\hline \hline Date (UT) & Exposure & Aperture size & Pixel size & \multicolumn{4}{c}{ Filter } \\
\cline { 5 - 8 } September, 1999 & $\mathrm{s}$ & arcmin & $\operatorname{arcsec}$ & Designation & $\lambda_{0}, \AA$ & $F W H M, \AA$ & Species \\
\hline 15.783 & 1200 & $7.8 \times 7.8$ & $1.0 \times 1.0$ & IF390 & 3894 & 105 & CN \\
15.783 & 1200 & $7.8 \times 5.2$ & $0.8 \times 0.8$ & RX & 6940 & 790 & Dust \\
15.801 & 1200 & $7.8 \times 7.8$ & $1.0 \times 1.0$ & IF390 & 3894 & 105 & CN \\
15.801 & 1200 & $7.8 \times 5.2$ & $0.8 \times 0.8$ & RX & 6940 & 790 & Dust \\
15.816 & 1200 & $7.8 \times 7.8$ & $1.0 \times 1.0$ & IF390 & 3894 & 105 & CN \\
15.815 & 900 & $7.8 \times 5.2$ & $0.8 \times 0.8$ & RX & 6940 & 790 & Dust \\
\hline
\end{tabular}

filter IF390 $\left(\lambda_{0}=389.4 \mathrm{~nm}, F W H M=10.5 \mathrm{~nm}\right)$ was used, while the broad-band filter RX $\left(\lambda_{0}=694 \mathrm{~nm}, F W H M=\right.$ $79 \mathrm{~nm}$ ) was chosen to study the light scattering by dust particles .

Details of the observed data are given in Table 1.

The original CCD frames were processed by standard procedures. They were bias subtracted, flat-fielded and cleaned of cosmic events. Star trails were detected and removed from the comet images using the Starfinder package (Diolaiti et al. 2000). The night sky level was estimated from the parts of the sky field not covered by the cometary coma and was subtracted. The standard stars 52 Her and 44 Boo have been observed as well. Their fluxes were taken from Voloshina et al. (1982).

Comparing blue and red images we found the same distributions of the detected emissions. We conclude that comet C/1999 J2 (Skiff) has no detectable CN atmosphere, and the well developed tail seeing in the red channel is purely made of dust. To increase the signal/noise ratio we summed the images obtained in the red channel and all the further results are specific to the summed frame with an effective exposure of $3300 \mathrm{~s}$. Additionally, as the comet image covers a small fraction of the CCD frame we extract a subframe of size $164^{\prime \prime} \times$ $164^{\prime \prime}$ and the following images, where the modelled results are compared with the observation data, have the same size. The appearance of the comet tail to be analyzed in the following sections is seen in Fig. 1.

\section{Model}

\subsection{Numerical details}

To determine the characteristics of the dust in the comet C/1999 J2 (Skiff) we developed a simple model combining the mechanical theory approach to calculate the trajectories of dust particles ejected from the nucleus and a Monte Carlo technique to determine beginning conditions of a particle under consideration. For the Monte Carlo technique, we already have experience in its practical implementation in cometary neutral atmospheres (Korsun 1995; Korsun \& Jockers 2002).

It is convenient to use the cometocentric frame of coordinates in our calculations. The coordinates $\xi$ and $\eta$ are in the comet orbit plane. The first of them, $\xi$, is positive radially outward from the Sun, and the second one, $\eta$, is directed opposite to the comet's motion along its orbit. The third coordinate, $\zeta$, is normal to the orbit plane. Related spherical coordinates $r, \theta$, and $\phi$ are used as well.

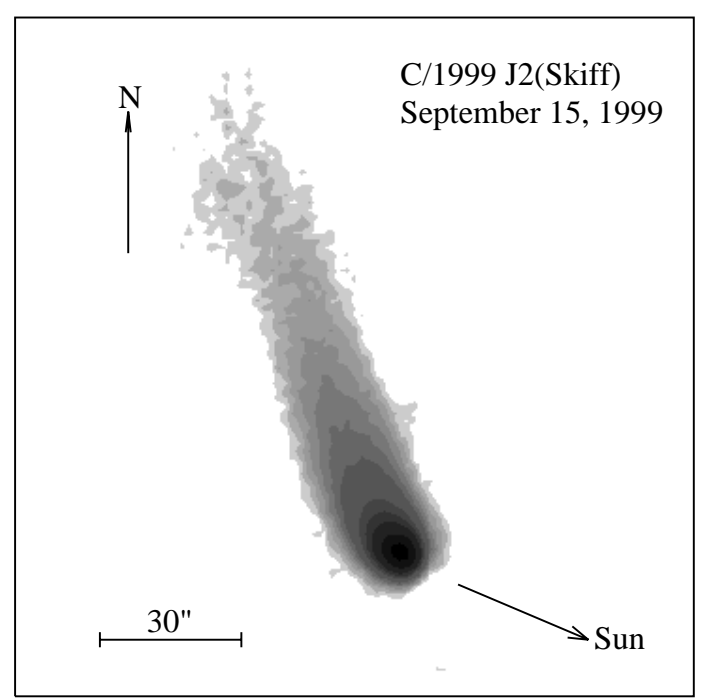

Fig. 1. The dust tail of comet C/1999 J2(Skiff). The size of the frame is $164^{\prime \prime} \times 164^{\prime \prime}$. The north is up.

To build the dust environment of the comet we trace the trajectories of many individual meteoroids ejected from the nucleus prior to the moment of observation. Initial geometric conditions of a particle leaving the nucleus are easily defined using Monte Carlo simulations. The fundamental equation of the method is as follows. If $p(x) \mathrm{d} x$ is the probability of $x$ lying between $x$ and $x+\mathrm{d} x$ and if it is known that $x$ always is in the range $a \leq x<b$, i. e. $\int_{a}^{b} p\left(x^{\prime}\right) \mathrm{d} x^{\prime}=1$ then a properly distributed random value of $x$ is found by solving

$R_{i}=\int_{a}^{x} p\left(x^{\prime}\right) \mathrm{d} x^{\prime}$

The random number $R_{i}$ is uniformly distributed in $\mathbf{0} \leq R_{i}<\mathbf{1}$.

According to the Monte Carlo principle the time when an individual dust particle is released from the nucleus, $t_{\mathrm{i}}$, can be determined from

$t_{\mathrm{i}}=R_{i}\left(T_{\mathrm{s}}-T_{\mathrm{o}}\right)$

Here $R_{i}$ is a random number uniformly distributed in $0 \leq R_{i}<1$, $T_{\mathrm{o}}$ is the time of observation, and $T_{\mathrm{S}}$ is the time when we start to build the dust tail. If the nucleus is an isotropic source of dust, the outflow direction of the particle has uniform probability. Then the spherical polar angles $\phi_{i}$ and $\theta_{i}$ can be calculated from

$\phi_{i}=2 \pi R_{i}, \quad \cos \left(\theta_{i}\right)=1-2 R_{i}$. 
Nonisotropic outflow of the cometary particles has been considered as well.

We treat the trajectory of each particle using computer program based on the Finson-Probstein method (Chörny 1986a; Chörny \& Sizonenko 1986b; Finson \& Probstein 1968). Its cometocentric coordinates are determined with the formulas

$r_{\mathrm{d}}=p_{\mathrm{d}}\left[1+e_{\mathrm{d}} \cos \left(\chi-\omega_{0}\right)\right]^{-1}$,

$e_{\mathrm{d}} \sinh (F)-F=n_{\mathrm{d}}\left(T_{\mathrm{o}}-T_{\mathrm{d}}\right)$.

The first of them is the equation of a trajectory of movement of the particle in the orbital cometary plane, and the second one is Kepler's equation for this particle. In these formulas the designations from the paper of Chörny (1986a) are used, namely, $r_{\mathrm{d}}$ is the heliocentric distance of the dust particle, $p_{\mathrm{d}}$ is the focal parameter of its orbit, $e_{\mathrm{d}}$ is its eccentricity, $\chi$ is its true anomaly, $\omega_{0}$ is the perihelion argument, $F$ is the eccentric anomaly of its orbit, $n_{\mathrm{d}}$ is its mean motion, and $T_{\mathrm{d}}$ is the moment of time at which the particle's perihelion is reached.

Assigning weight coefficients $w$ to the counted particles makes our model more realistic. The heliocentric dependence of the dust production rate $Q_{\mathrm{d}}$ is controlled by $w_{r}=r^{-n}$, where $n$ is a model parameter. Additionally, the heliocentric dependence of the ejection velocities of the dust particles is controlled by $w_{r}=r^{-0.5}$.

In order to determine the coordinates of the dust grains in the sky plane the equations derived by Finson \& Probstein (1968) have been used. As a result we have locations of the individual dust particles in the modelled atmosphere at the moment of observation. To build a model dust atmosphere with satisfactory statistics we trace $10^{7}$ particles in our calculations.

\subsection{Physical assumptions}

The computational scheme described above needs to be added to possible physical processes occurring in the case of the comet C/1999 J2 (Skiff).

The comet was observed at the heliocentric distance of 7.24 AU. It is well known that water ice sublimation begins near $180 \mathrm{~K}$. Having low albedo, the cometary nucleus can reach this equilibrium temperature near $r=5-6 \mathrm{AU}$, thus a volatile other than $\mathrm{H}_{2} \mathrm{O}$ is responsible for the dust tail formation. It is expected that this supervolatile drives off grains of water ice contaminated by small refractory inclusions at very large heliocentric distances. Analyzing narrowband photometry of 85 comets, A'Hearn et al. (1995) concluded that in all comets with large perihelia there is a halo of relatively pure icy grains driven off by a relatively volatile species with the grains expanding very slowly. Evidence for water ice grains has been obtained in the comae of several distant comets (Campins et al. 1983; Davies et al. 1997; Hanner 1983; Lellouch et al. 1998).

The motion of a dust particle outside the collision zone is controlled by the ratio of two forces, namely, the solar gravity attraction $F_{\mathrm{G}}$ and the force $F_{\mathrm{R}}$ caused by solar radiation pressure:

$\beta=\frac{F_{\mathrm{R}}}{F_{\mathrm{G}}}=0.57 \times 10^{-4} \frac{Q_{\mathrm{pr}}}{\rho a}$.
Here $\rho$ is the mass density, $a$ is the radius of a dust particle, $\rho$ and $a$ are expressed in CGS units. $\rho=1 \mathrm{~g} \mathrm{~cm}^{-3}$ is assumed as it is expected that the dust particles in the tail are dirty waterice ones. $Q_{\mathrm{pr}}$ is the efficiency factor for radiation pressure and is equal to 1 when the dust particles are large compared to the wavelength of sunlight. So, Eq. (7) can be reduced to

$\beta=0.57 a^{-1}(a$ in $\mu \mathrm{m})$.

In the proposed model we do not consider the gas-dynamic dragging of the dust particles in the collision gas coma. It is more convenient to deal with ejection velocity $v_{\mathrm{e}}=v(a)$ of a particle of radius $a$, i.e. of the velocity attained by a grain far enough from the nucleus, where its interaction with the gas has become negligible. Using gas-dynamical theory, Sekanina et al. (1992) derived an expression

$v(a)=\frac{1}{A_{0}+B_{0} \sqrt{a}}$.

Here $A_{0}$ and $B_{0}$ generally depend upon the sublimation rate, the thermal gas velocity, the nucleus mass, and the dust/gas mass ratio.

For the numerical calculations we adopt the modified Sekanina's relation (Sekanina 1992b) derived for the dust environment of the distant comet $\mathrm{P} / \mathrm{Sch}$ wassmann-Wachmann case:

$v(\beta)=\frac{A_{1}}{0.12+\beta^{-0.5}} r^{-0.5}$.

Here the $\beta$ dependence of the ejection velocity $v(\beta)$ is used, instead of $v(a)$, with $\beta$ from Eq. (8). The commonly used heliocentric distance dependence of $v_{\mathrm{e}} \sim r^{-0.5}$ and an additional numerical coefficient $A_{1}$ are incorporated as well. The value of $A_{1}$ is determined in the model runs.

As to the particle size distribution, we use commonly accepted power law with power exponent $k$ as a model parameter $\mathrm{d} n \sim a^{k} \mathrm{~d} a$.

It is known that cometary particles are fluffy and non-spherical. Methods to study complex particle exist. One can find a detailed review of a variety of approximations in Mishchenko et al. (2000). Unfortunately, very little is known about the structure of the dust aggregates in a cometary tail. Also there are numerical limitations regarding large particle treatment. The scattering properties of the cometary dust are mostly based on the simplified but well developed Mie theory. Mie theory, i.e. the theory describing the scattering of light at spherical particles, is the only readily available scattering theory, especially if a model with many free parameters is used. To calculate the scattered light from the modelled dust tail we choose the Mie theory as well.

Recent spacecraft and groundbased observations give strong evidence that most of the nuclear surface is crusted over and a few isolated spots are active and contribute the main fraction of the observed dust and gas (Britt et al. 2002; Keller et al. 1987). Since the spin period of the comet nucleus is commonly much shorter, hours, relative to the period of dust tail formation, months and years, an approximation of the Sun-facing coma is very useful. The dust is assumed to be ejected into a cone with its axis toward the Sun. Here the half width of the cone $\omega$ is used as a model parameter. 


\subsection{Sample runs}

To fit the observed dust tail it is useful to know in what way each involved model parameter transforms the final dust tail appearance. As a reference sample we use the modelled tail successfully fitting our observations (Fig. 2a). The related model parameters one can find in Table 2. The reference dust tail is calculated for the comet C/1999 J2 (Skiff) and is shown in the cometary orbit plane. As the appearance of the tail in the cometary orbit plane is expected to be much more spread out, compared to its projection on the sky plane, the size of each modelled frame is adopted to be $13.3 \times 13$.3. Because of limited model statistics the image was convolved with a 2D-Gaussian, $F W H M=2$ '. 5 , to improve its appearance. The other images in Fig. 2 are the same as the first one, except for the model parameters indicated on the top of each following image. It was found that these parameters have a dramatic effect on the resulting appearance of the calculated dust tail.

The younger the particles involved in simulating the dust tail, the less spread is the tail appearance. This is clearly seen in Fig. 2c. In contrast, the older particles tend to occupy a more spread area (see Fig. 2b). The dust tail length and its orientation strongly depend on the age of the dust. The width of the dust tail is controlled by the dust particle ejection velocity. Figure $2 \mathrm{e}$ shows the significant tail widening when the ejection velocity is enlarged to $v_{\mathrm{e}}^{\prime}=1.5 v_{\mathrm{e}}$.

The power index of the dust size distribution controls the departure of the tail direction from the $\xi$ axis and fractional populations of the particles of different size along the tail. The higher the power index the more abundant are the light particles and the angle between the tail direction and $\xi$ axis is less (see Fig. 2d). When we remove the fraction of the lighter particles from our model calculation, the tail tends to be shorter and the angle between the tail direction and $\xi$ axis becomes greater. Figure $2 \mathrm{f}$ refers to this case.

\section{Results and discussion}

There are a number of comets exhibiting quite significant activity at heliocentric distances sufficiently large to be explained by water sublimation. Some of them also have tails of distinctive appearance at great distances from the Sun. The tails are featureless, without appreciable broadening with increasing distance from the nucleus, and the length of the tail is several minutes of an arc from the well-condensed head (Roemer 1962). Sekanina $(1973,1975)$ was the first to interpret the orientation and general profile of the tails at large heliocentric distances using an approach developed by Finson \& Probstein (1968). The tail was successfully covered by a set of synchrones associated with heavy dirty ice grains with extremely low repulsive accelerations.

Currently, well-developed models of cometary dust environment formation have been initiated by Fulle (1987) and Sekanina (1992b). However, these have been applied to analyze the burst events in the case of distant comet activity, while we fit the tail resulting from non-burst outflow of gas and dust from the nucleus.
Table 2. Derived parameters for the comet C/1999 J2 (Skiff) tail.

\begin{tabular}{ll}
\hline \hline Parameter & Value \\
\hline Power exponent ${ }^{a}, k$ & -3.5 \\
Range of $a, a_{\min }-a_{\max }$ & $10 .-800 . \mu \mathrm{m}$ \\
Ejection velocities, $v_{\mathrm{e}}$ & $10.7^{b}-1.2^{c} \mathrm{~m} \mathrm{~s}^{-1}$ \\
Maximum age of the particles & 540 days \\
Heliocentric dependence of $Q_{\mathrm{d}}$ & $r^{-2}$ \\
Heliocentric dependence of $v_{\mathrm{e}}$ & $r^{-0.5}$ \\
Half width of the cone, $\omega$ & $90^{\circ}$ \\
Angular anisotropy of $Q_{\mathrm{d}}$ & $U^{d}(50 \%)+S^{e}(50 \%)$ \\
\hline
\end{tabular}

${ }^{a}$ In the particle size distribution law, $a^{k}$.

${ }^{b}$ For $a=10 \mu \mathrm{m}$ at $r=7.24 \mathrm{AU}$.

${ }^{c}$ For $a=800 \mu \mathrm{m}$ at $r=7.24 \mathrm{AU}$.

${ }^{d} U=$ Fraction of the dust particles ejected isotropically.

e $S=$ Fraction of the dust particles ejected from sunlit side of the nucleus.

The dust tail of the comet C/1999 J2 (Skiff) has been fitted using the model described in the previous section. Table 2 lists the parameters involved in the model runs. Figure 3 shows the observed tail isophotes and modelled ones. It is clearly seen that the modelled tail appearance represents the characteristic features of the dust tails observed at large distances from the Sun, such as fairly well defined boundaries, structureless and a slight curvature of the tails, the absence of their appreciable broadening with increasing distance from the nucleus. Three surface profiles across the tail and one approximately along the tail serve as an additional control of the fitting process.

The well-developed dust tail of the comet C/1999 J2 (Skiff) has been observed at the heliocentric distance of 7.24 AU.

Water does not sublimate at this and greater heliocentric distances and cannot be responsible for the dust tail formation. $\mathrm{CO}$ and $\mathrm{CO}_{2}$ ices are good candidates, because of their large abundance and low vaporization temperatures (Sekanina 1992a). Recent observations show that CO is an important outgassing species in distant comets and can serve as a driver for the observed grains (Biver et al. 1996; Gunnarsson et al. 2002; Jewitt et al. 1996; Senay \& Jewitt 1994). If the sublimation is the main mechanism of the observed tail formation, the particles ejected at the heliocentric distances greater than, for example, 10. AU must be present in the tail.

Our model calculations show that the oldest particles forming the detectable tail are as old as only 540 days, that is are ejected at the heliocentric distance of 8.6 AU. Figure 4 represents some arguments in favor of this result. In this figure we show modelled locations of the particles having a size of $0.8 \mathrm{~mm}$ and being ejected at $8.6 \mathrm{AU}, 10 \mathrm{AU}$, and $12 \mathrm{AU}$, that is 540,900 , and 1180 days prior to the moment of the observations. The loci of the particles emitted at the heliocentric distance of $8.6 \mathrm{AU}$ are within the observed tail, while ones emitted at larger heliocentric distances tend to leave the tail. Considering involved particles as dirty ice ones, it is impossible, varying the model parameters, to obtain the result, when the particles emitted at the heliocentric distances larger than 8.6 AU would be placed within the tail. 


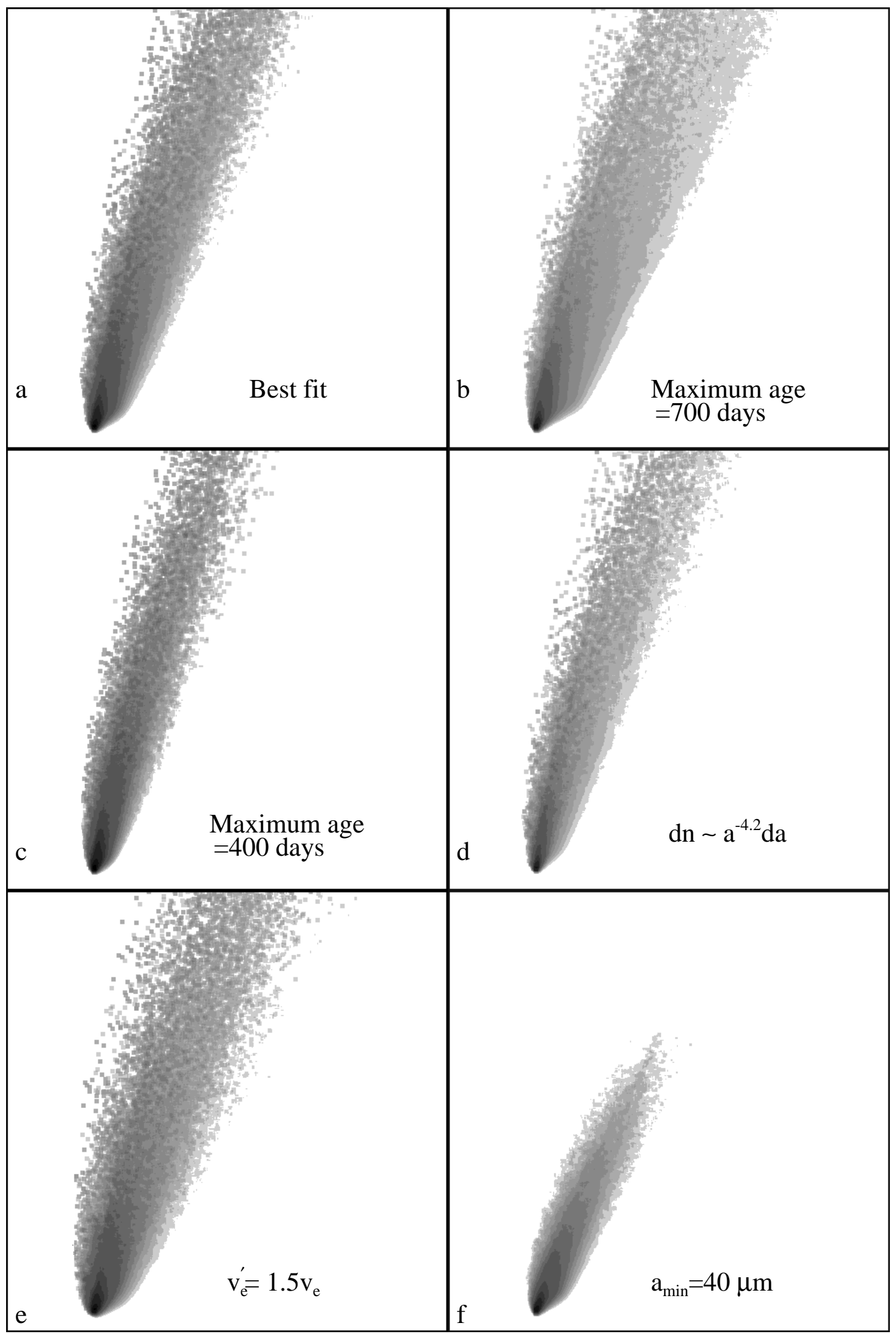

Fig. 2. a) Monte Carlo simulation of dust tail based on the set of parameters listed in Table 2. The other images, from b) to f), in this figure represent the same simulations as a), except for the parameters indicated on each following image. The tail appearance is shown in the cometary orbit plane. The images have size of $13.3 \times 13.3$ and are smoothed to $2 . ' 5$ to improve their appearance. 


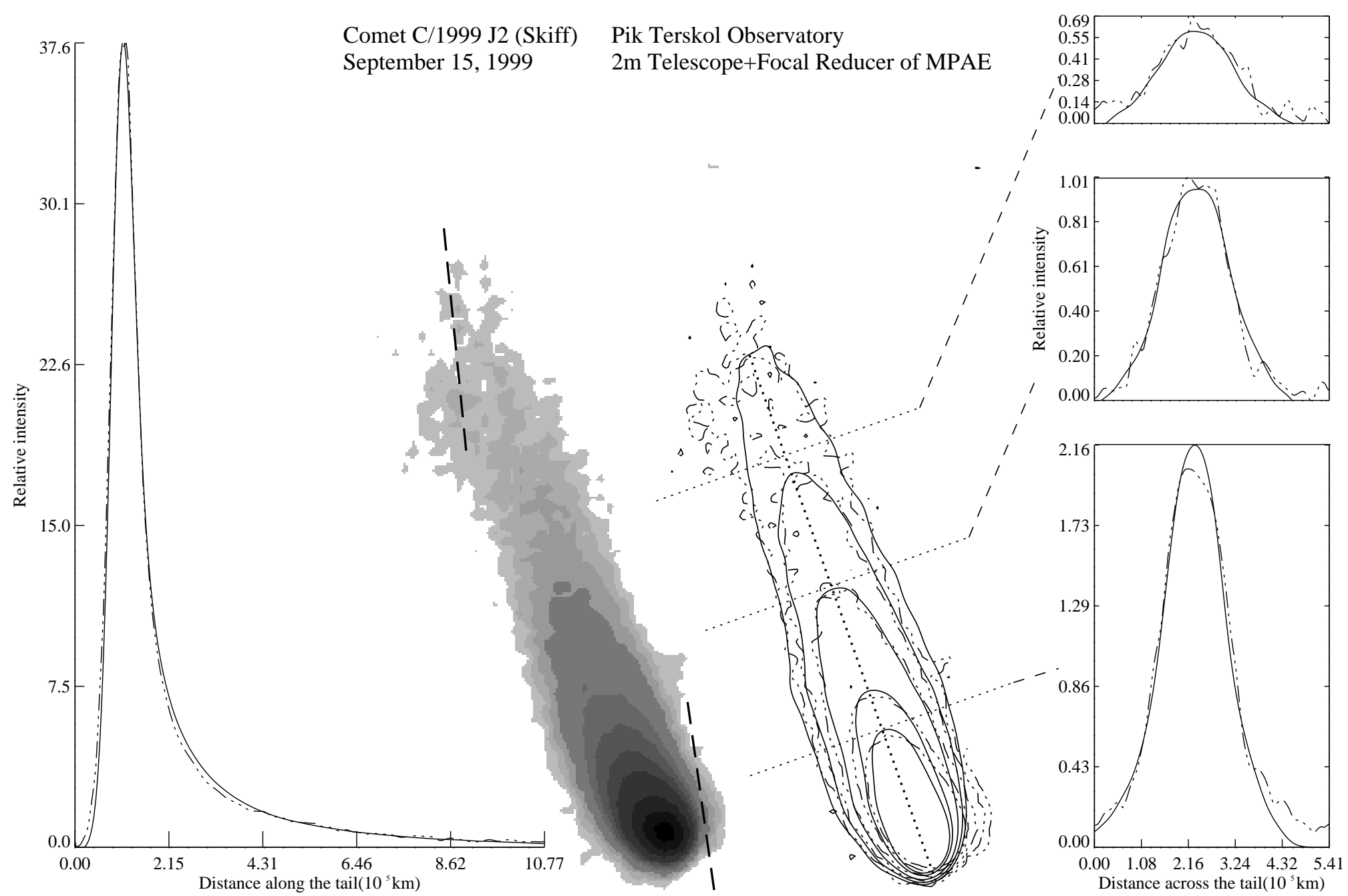

Fig. 3. Observed tail of the comet C/1999 J2 (Skiff) as well as modelled and observed isophotes and profiles of the dust tail. Because of curvature of the tail the profiles displayed on the left side of the picture are extracted approximately along the tail. The transverse profiles displayed on the right side of the picture are extracted at the distances of $2.8 \times 10^{5} \mathrm{~km}, 5.2 \times 10^{5} \mathrm{~km}$, and $7.4 \times 10^{5} \mathrm{~km}$ from the brightest point. All the profiles are averaged across a lane of $5^{\prime \prime}$ width. Bold-dashed lines superimposed on the tail image indicate the star trails in the original image. Because the procedure of trail removal is not ideal, we have uncompensated blends in the analyzed tail. 


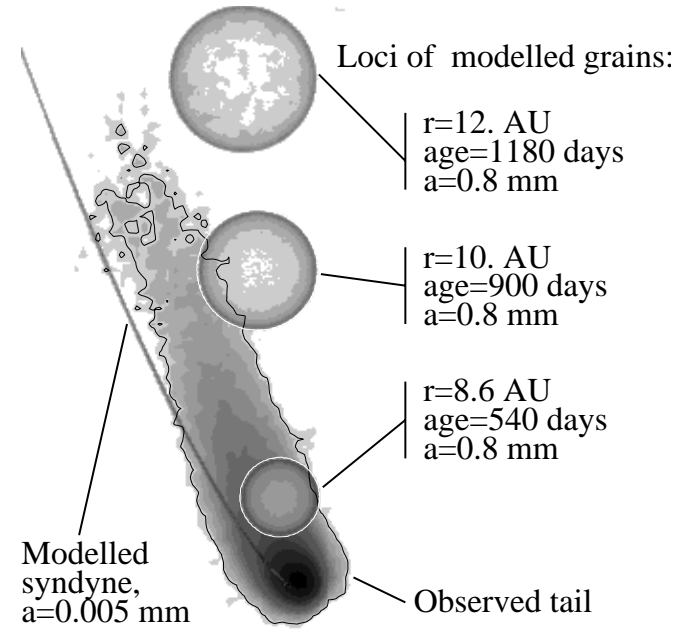

Fig. 4. Modelled envelopes of the dust particles being superimposed on the observed tail. All the particles in the envelopes have the same size of $0.8 \mathrm{~mm}$ and were emitted at three discrete moments prior to the moment of observation. Modelled syndyne for particles having size of $0.005 \mathrm{~mm}$ is shown as well.

So, we conclude that there is not sufficient exposed $\mathrm{CO}$ and $\mathrm{CO}_{2}$ ice on the surface of the comet $\mathrm{C} / 1999 \mathrm{~J} 2$ (Skiff) nucleus to form the detected tail by sublimation only, and we have to look for another mechanism of tail formation.

The phenomenon of phase transition from amorphous to crystalline ice, taken into account in recently published sublimation models to explain intensification of cometary activity at large distances from the Sun (Capria 2002; Prialnik 2002), can resolve the problem. The reaction, being exothermic, results in the release of a large amount of CO initially trapped in amorphous ice. According to the numerical calculations of Prialnik \& Bar-Nun (1992) the crystallization of amorphous ice is significantly activated at heliocentric distances between 5 and $17 \mathrm{AU}$, and our results are consistent with these data. So, the phenomenon described here can be responsible for the formation of the tail in the comet C/1999 J2 (Skiff).

As it was mentioned in the previous section, it seems to be natural to consider the dust particles as water ice ones contaminated by dirty inclusions (Mukai et al. 1989). Observational evidence for a water ice halo has been obtained from investigations of the continuum spectra of the comet C/1995 O1 (Hale-Bopp) at large heliocentric distances (Davies et al. 1997; Lellouch et al. 1998).

Assuming $Q_{\mathrm{pr}}=1$ and $\rho=1 \mathrm{~g} \mathrm{~cm}^{-3}$ for dirty ice grains, we derive their minimum radius to be $a_{\min }=0.01 \mathrm{~mm}$ and maximum one to be $a_{\max }=0.8 \mathrm{~mm}$. The modelled syndyne for particles having a size of $0.005 \mathrm{~mm}$ is not placed along the observed tail, which illustrates that particles with sizes less than $0.01 \mathrm{~mm}$ do not determine the appearance of the observed tail. Whereas the upper limit of the observed particles is determined by the production of the cometary gas, the lower one needs to be clarified. The particles having a size smaller than $0.01 \mathrm{~mm}$ can be optically inactive or, more realistically, they are in significant deficit in the cometary tail owing to sublimation of water ice. Indeed, Mukai (1986) argues that the lifetime of dirty ice grains at a heliocentric distance of $7 \mathrm{AU}$, having a radius greater than $0.01 \mathrm{~mm}$, is over $10^{8}$ days, while when the radius of the ice particle is varied from 0.01 to $0.001 \mathrm{~mm}$ its lifetime is dramatically decreased to a few hours. The dust tail of the comet C/1995 O1 (Hale-Bopp) observed at large heliocentric distances was fitted assuming the minimum size of the dust grains to be at least several microns (Fulle et al. 1998). The dust environments of the comets $\mathrm{P} / \mathrm{Halley}$ $(r \sim 14 \mathrm{AU})$ and $\mathrm{P} /$ Schwassmann - Wachmann $1(\mathrm{P} / \mathrm{SW} 1)(r \sim$ $6 \mathrm{AU})$ have been modelled involving micron- and submicronsized grains (Sekanina 1992b; Sekanina et al. 1992), that differs from our choice. In our opinion, these cases were outburst events. Therefore, the dust environments were formed in a short period of time and the small particles were not removed due to sublimation.

The dust size distribution in our simulations is referred to the particles leaving the nucleus, not to the particles occupying the tail. The power index of our dust size distribution is used in the same sense as in the inverse Monte Carlo model developed by Fulle (1987) and was applied to many comets. Its values for the distant comets ranged between -3.2 and -3.6 (Fulle et al. 1998). Sekanina (1992b) and Sekanina et al. (1992) give values $-3.7(\mathrm{P} /$ Halley) and $-3.0(\mathrm{P} / \mathrm{SW} 1)$ for grains comparable in size with our ones. Thus, our power index, being equal to -3.5 , is in a good agreement with these results.

Since radiation pressure depends on particle size, cometary grains are sorted according to size and different parts of the dust coma and dust tail tend to have different size distributions. This effect can be easily demonstrated using model runs. In Fig. 5a one can see the modelled image which is the best fit to our observations. Figures $5 b-d$ show that dust particles having different sizes occupy different regions in the cometary tail. Lighter particles tend to be close to the $\xi$ axis and travel far from the nucleus, while larger ones have a larger deflection from the $\xi$ axis and concentrate closer to the nucleus. Two isophotes associated with the image in Fig. 5a are also superimposed on the images in Figs. 5b-d to clarify this effect.

The dynamical study of dust particles in distant comets suggests large grains and low dust velocities (Meech 1992; Meech \& Farnham 1997; Meech \& Jewitt 1987; Sekanina 1982). We have a similar result. Using Eqs. (8) and (10) with the derived coefficient $A_{1}$ equal to 120 , one can find that for $a_{\mathrm{min}}=$ $0.01 \mathrm{~mm}$ and $r=7.24$ AU the terminal velocity is $10.7 \mathrm{~m} \mathrm{~s}^{-1}$ and for $a_{\max }=0.8 \mathrm{~mm}$ it is $1.2 \mathrm{~m} \mathrm{~s}^{-1}$.

Our model runs show that the sunlit side of the nucleus is more preferable for the release of dust. Emission by the cometary head is fitted assuming that $50 \%$ of the dust particles are released isotropically from the nucleus, while the remaining $50 \%$ of them are released from the sunlit side (half width of the cone $\omega$ is $90^{\circ}$ ). Note that attempts to fit the tail by isotropic dust emission only make our model fit poorer.

We note that the detailed dust-dynamical modelling of the tail of the distant comet C/1999 J2 (Skiff) agrees with dynamical study conclusions for other distant comets.

Acknowledgements. We thank Professor K. Jockers (Max-PlankInstitute for Aeronomy, Germany) and the personnel of the Pik Terskol Observatory for help in making these observations. We also thank Professor K. Jockers for critical comments that resulted in significant improvements to the paper. We are grateful to the referee 

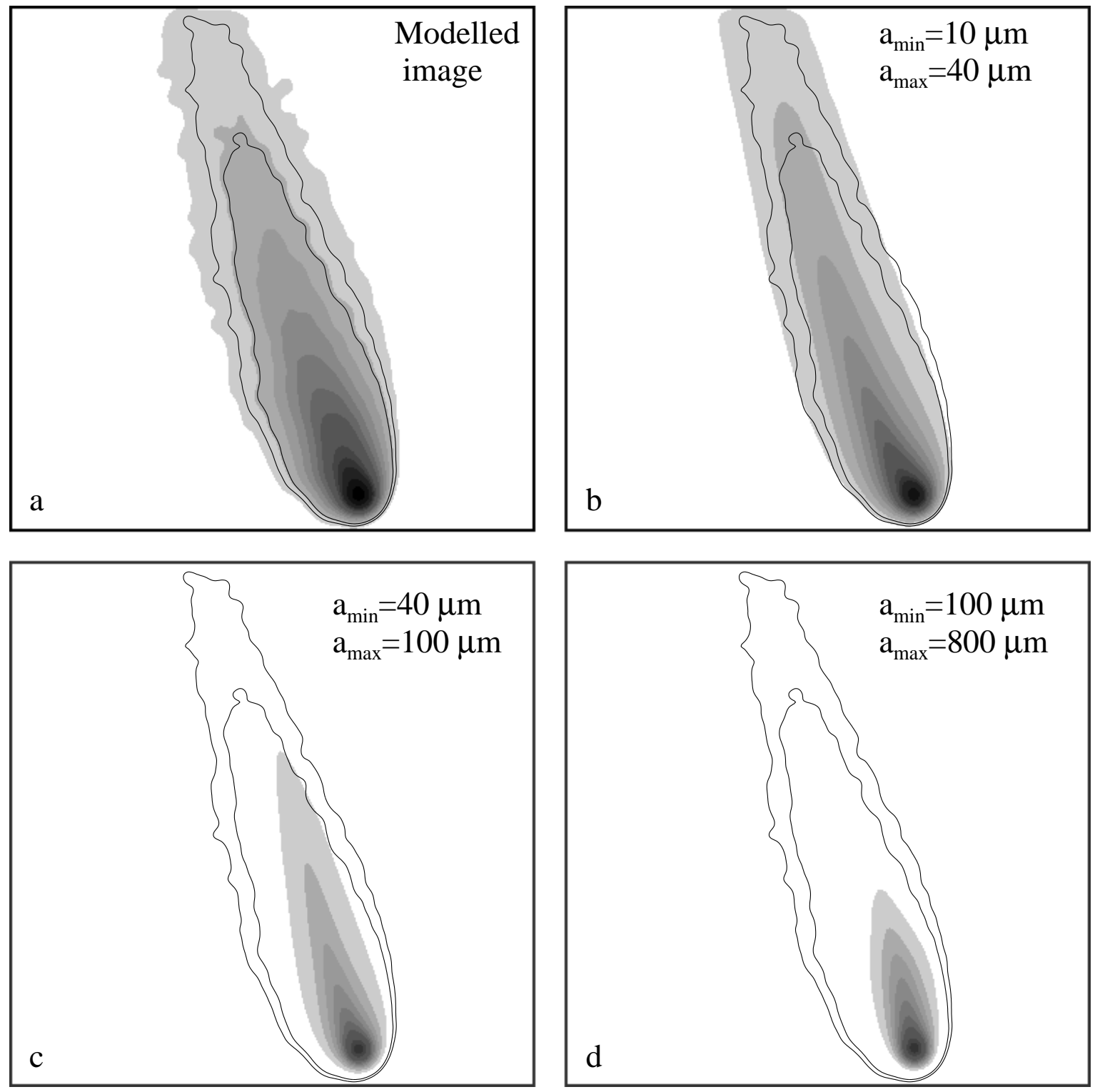

Fig. 5. The abundance of the different size particles along the modelled tail. Image a) shows the most probable fit to the observed tail. Images b)-d) show that particles in various sizes occupy different areas in the dust tail suggesting different dust size distribution along the tail.

Dr. J. C. Brandt for his careful reading of the manuscript and useful comments as well.

\section{References}

A’Hearn, M. F., Millis, R. L., Schleicher, D. G., Osip, D. J., \& Birch, P. V. 1995, Icarus, 118, 223

Biver, N., Rauer. H., Despois, D, et al. 1996, Nature, 380, 137

Britt, D. T., Boice, D. C., Buratti, B. J., et al. 2002, LPI, 33, 1686

Campins, H., Rieke, G. H., \& Lebofsky, M. J. 1983, Nature, 302, 405

Capria, M. T. 2002, Earth Moon \& Planets, 89, 161

Chörny, G. F. 1986a, Kinem. Phys. Cel. Bod., 2(2), 60

Chörny, G. F., \& Sizonenko, Yu. V. 1986b, Kinem. Phys. Cel. Bod., 2(3), 59

Davies, J. K., Roush, T. L., Cruikshank, D. P., et al. 1997, Icarus, 127, 238

Diolaiti, E., Bendinelli, O., Bonaccini, D., et al. 2000, A\&A, 147, 335
Finson, M. L., \& Probstein, R. F. 1968, ApJ, 154, 353

Fukushima, T., Nakajima, T., \& Watanabe, J. 2000, IAU Circ., 7415

Fulle, M. 1987, A\&A, 171, 327

Fulle, M. 1992, Nature, 217, 293

Fulle, M. 1994, A\&A, 282, 980

Fulle, M., Cremonese, G., \& Bohm, C. 1998, ApJ, 116, 1470

Gunnarsson, M., Rickman, H., Festou, M. C., et al. 2002, Icarus, 157, 309

Hanner, M. S. 1983, AJ, 277, L75

Jewitt, D., Senay, M., \& Matthews, H. 1996, Science, 271, 1110

Jockers, K. 1997, Exper. Astron., 7, 305

Keller, H. U., Delamere, W. A., Huebner, W. F., et al. 1987, A\&A, 187,807

Korsun, P. P. 1995, Astron. Nachr., 4, 255

Korsun, P. P., \& Jockers, K. 2002, A\&A, 381, 703

Lellouch, E., Crovisier, J., Lim, T., et al. 1998, A\&A, 339, L9

Meech, K. J. 1992, in the Workshop on the Activity of Distant Comets, ed. W. F. Huebner, H. U. Keller, D. Jewitt, J. Klinger, \& R. West, 12 
Meech, K. J., \& Farnham, T. L. 1997, M\&PSA, 32, 89

Meech, K. J., \& Jewitt, D. 1987, Nature, 328, 506

Mishchenko, et al. 1990, Light Scattering by Nonspherical Particles, ed. M. I. Mishchenko, J. W. Hovenier, \& L. D. Travis (Academic Press)

Mukai, T. 1986, A\&A, 164, 397

Mukai, T., Mukai, S., \& Kikuchi, S. 1987, A\&A, 650

Mukai, T., Fechtig, H., Grun, E., \& Giese, R. H. 1989, Icarus, 80,

Prialnik, D. 2002, Earth Moon \& Planets, 89, 27

Prialnik, D., \& Bar-Nun, A. 1992, in the Workshop on the Activity of Distant Comets, ed. W. F. Huebner, H. U. Keller, D. Jewitt, J. Klinger, \& R. West, 100

Roemer, E. 1962, PASP, 74, 351

Sekanina, Z. 1973, ASTLAI, 14, 175

Sekanina, Z. 1975, Icarus, 25, 218

Sekanina, Z. 1982, AJ, 87, 161
Sekanina, Z. 1992a, ACM 1991, Lunar and Planetary Institute, Houston, 545

Sekanina, Z. 1992b, in the Workshop on the Activity of Distant Comets, ed. W. F. Huebner, H. U. Keller, D. Jewitt, J. Klinger, \& R. West, 166

Sekanina, Z., Larson, S. M., Hainaut, O., Smette, A., \& West, R. M. 1992, A\&A, 263, 367

Senay, M. C., \& Jewitt, D. 1994, Nature, 371, 229

Skiff, B. A., Koehn, B. W., Tichy, M., \& Moravee, Z. 1999, IAU Circ., 7165

Shanklin, J. 2001, The Comet's Tale, 8(2), 15

Szabo, Gy., Csak, B., Sarneczky, K., \& Kiss, L. L. 2001, A\&A, 374, 712

Voloshina, I. B., Glushneva, I. N., Doroshenko, B. T., et al. 1982, Spectrophotometrija yarkih zvezd (Moscow: Nauka) 